\title{
La familia Carvia Bernal y la Facultad de Medicina. Una historia de librepensamiento y masonería en el Cádiz de finales del siglo XIX
}

\author{
Manuel Almisas Albéndiz \\ Licenciado en Medicina por la Facultad de Medicina de Cádiz \\ y profesor jubilado de Enseñanza Secundaria
}

\begin{abstract}
RESUMEN: No es muy conocido que numerosos médicos gaditanos ingresaron en alguna de las muchas logias masónicas que existieron en Cádiz en el último tercio del siglo XIX. Pero menos aún ha trascendido que la familia del portero de la Facultad de Medicina, Manuel Carvia Carneiro, que llegó a ser bedel y finalmente conserje, tuviera una relación tan estrecha con la masonería gaditana, hasta tal punto que sus hijas Amalia y Ana fundaron en abril de 1895 la única logia femenina que ha existido en Cádiz.
\end{abstract}

Palabras clave: Facultad de Medicina, Masonería, Masonería femenina.

\section{The Carvia Bernal family and the School of Medicine -A story of freethinking and freemasonry in the Cadiz of the late nineteenth century-}

ABSTRACT: It is not well known that many doctors entered some of the many masonic lodges that existed in Cadiz in the last third of the nineteenth century. But even less has it leaked out that the family of the doorman of the Faculty of Medicine, Manuel Carvia Carneiro, who became janitor and finally concierge, had such a close relationship with the Freemasonry of Cadiz, to such an extent that his daughters Amalia and Ana founded in April 1895 the only female lodge that has ever existed in Cadiz.

Keywords: School of Medicine, Freemasonry, Female Freemasonry.

Es ampliamente conocida la proliferación de logias masónicas en el Cádiz del siglo XIX, sin duda la mayor de Andalucía, especialmente desde la Restauración borbónica y ya con una hegemonía aplastante a partir de $1887^{1}$, pero no lo es tanto su extensión entre la clase médica de Cádiz y mucho menos su estrecha relación con una familia que mantuvo una dilatada relación laboral con la Facultad de Medicina, la familia Carvia Bernal. Esta es su historia.

1 ENRÍQUEZ DEL ÁRBOL 1987.

DOI del artículo:

https://doi.org/10.25267/Cuad investig fondos arch UCA.2020.i2.01 
Cuando en 1875 Manuel Carvia Carneiro, de origen gallego, entró a trabajar como portero para la Facultad de Medicina y Cirugía de la Universidad Literaria de Sevilla, quizá no se imaginaba que su vida iba a estar ligada hasta el final de sus días a esa ilustre institución gaditana. Manuel ocupó la casa-portería de la Facultad situada en la entonces plaza del Rey Alfonso XII, a donde se mudó con su mujer Ana Bernal Ramos y sus tres hijos adolescentes: Amalia, la mayor de 14 años, Manuel de 12 y Ana de 10. Desde que el matrimonio se casó en 1861 en la Parroquia Castrense cuando vivían en la cercana calle del Hércules, siempre residieron en el mismo barrio y sus tres hijos fueron bautizados en dicha Parroquia, por lo que es más que probable que Manuel hubiera trabajado hasta entonces como simple «peón» en el Hospital Real o en el Hospital Civil Provincial, ambos situados junto a la Facultad de Medicina, o en la misma Facultad, que empleaba entonces a tres mozos para distintas tareas, incluido el encargado del mantenimiento del anfiteatro de anatomía.

En 1883, después de llevar ocho años trabajando para la institución, Manuel tuvo que conseguir algún tipo de beca o ayuda para hijos de trabajadores de la Facultad, porque su hijo mayor se matriculó en el curso preparatorio de la Facultad de Medicina ${ }^{2}$, carrera impensable para una familia tan humilde. En ese momento Manuel estaba terminando el servicio militar y su padre fue el que firmó las solicitudes de matrícula. Como su padre firmó toda su vida «Manuel Carbia», los primeros documentos oficiales de su hijo también constan como «Manuel Carbia Bernal».

Manuel, hijo, había estudiado la enseñanza primaria en la Escuela Práctica de niños de la Normal de Maestros de Cádiz, en la calle de las Bulas, donde se respiraba un ambiente progresista, republicano y masón en algunos de sus maestros y responsables de la escuela, como José María Franco, y José María Rioseco, además del director de la misma el también republicano Hermengaudio Cuenca Arias. Y tuvo que destacar académicamente y ser bien considerado por sus maestros pues de allí pasó al Instituto de Enseñanza secundaria, donde el profesorado de bachillerato no era menos demócrata, como los destacados seguidores de las doctrinas filosóficas y pedagógicas de Karl Krause, los licenciados Alfonso Moreno Espinosa y Romualdo Álvarez Espino. Así que es indudable que Manuel Carvia Bernal, al ingresar en la Facultad de Medicina, ya podría tener unas inquietudes muy cercanas a la masonería, el librepensamiento y el republicanismo, ideas todas ellas que en aquella época estaban muy interrelacionadas. Aunque quizá, ni siquiera hubiera hecho falta ese aprendizaje y ese contacto previo, pues la propia Facultad era un «hervidero», usando la misma palabra que la profesora Fernández ${ }^{3}$, de estudiantes y profesores imbuidos de principios cercanos a la masonería, y numerosos médicos de Cádiz pertenecían a las diversas logias masónicas establecidas en la ciudad.

2 Archivo de la Universidad de Cádiz (AUCA). C-147 (2)-29 FM.

3 FERNÁNDEZ LUCEÑO, M. ${ }^{a}$ V. Médicos republicanos y masones en Andalucía contemporánea. La represión franquista. Ed. Aconcagua, 2016.

DOI del artículo:

https://doi.org/10.25267/Cuad investig fondos arch UCA.2020.i2.01 
Figura 1. Carátula del expediente de Manuel Carvia Bernal

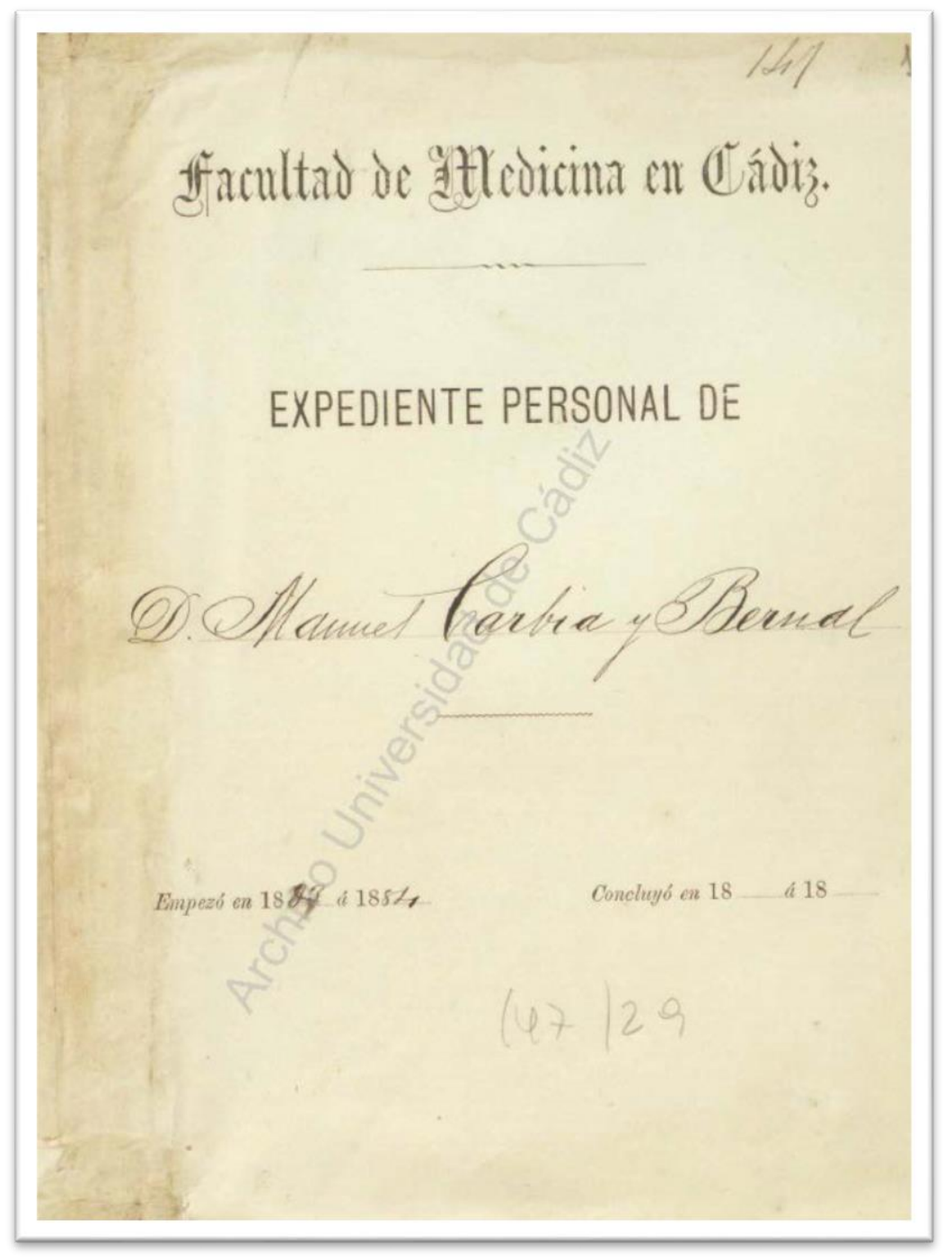

Fuente: Archivo de la Universidad de Cádiz (AUCA)

Es un tema muy poco estudiado, pero solo analizando los «Cuadros de obreros» de las logias «Regeneración» ${ }^{4} \mathrm{y}$ «Pirámides» ${ }^{5}$ de los años 1880 a 1885 se comprueba que la relación de médicos en las mismas era numerosa, destacando Arturo Monje Cuadrado («Arturo» de nombre simbólico) en la Logia «Regeneración n. ${ }^{\circ} 88 »$, de la que fue Venerable Maestro y Juan de Dios Delgado Serrano («Chommel» de nombre simbólico) que llegó a ser también Venerable Maestro de la Logia «Pirámides 250», y retengamos que como «miembro de honor» de esta última logia figuró algunos años el famoso médico Cayetano del Toro y Quartiellers. Sin contar los posibles estudiantes de Medicina que solo venían en los listados como «estudiantes», sin especificar.

4 Archivo del Centro Documental de la Memoria Histórica (CDMH) de Salamanca. Expediente 1, Legajo 472-A.

5 Archivo del Centro Documental de la Memoria Histórica (CDMH) de Salamanca. Expediente 5, Legajo 741-A. 


\section{La familia Carvia Bernal y la Facultad de Medicina. Una historia de librepensamiento y masonería en el Cádiz de finales del siglo XIX}

Así que no puede sorprender en absoluto que, en abril de 1887, cuando ya llevaba cuatro años estudiando la carrera, Manuel Carvia Bernal fuera iniciado en los ritos masónicos de la Logia «Regeneración». Sí es más llamativo, por el contrario, que tras de sí, al mes siguiente, arrastrara a sus dos jóvenes hermanas, Amalia y Ana, que se convertirán en las primeras mujeres de dicha logia gaditana, junto a Dolores Guillón y Juana Varo, que se iniciaron el mismo día del mes de mayo de 1887.

En ese momento, Amalia, de 26 años de edad, decía ser «pintora» y Ana «Estudiante» de 21 años recién cumplidos, ambas solteras y los tres hermanos afirmaban vivir en la calle «Facultad de Medicina, $n .^{\circ} 0 »$. Así que desde ese mes de mayo de 1887 y durante algunos años más, en la casa-portería de la Facultad vivieron tres jóvenes masones, llegando también hasta ese lugar más apartado y doméstico el ambiente masón que se respiraba en las aulas y en los despachos y pasillos de la Facultad.

Amalia no había cursado estudios más allá de los elementales pero sí asistió a clases de pintura sin acabarlos, por lo que se atrevió a decir que tenía esa profesión, mientras Ana Carvia Bernal estaba terminando los estudios de Bachillerato en el Instituto de la calle San Francisco, y había formado parte de un reducido grupo de mujeres que se matricularon en el Instituto de Secundaria en el curso 1881-1882, justo antes de que entrara en vigor una nueva restricción que impedía a las mujeres cursar estudios superiores ${ }^{6}$.

La trayectoria de los tres hermanos en la masonería fue al principio muy semejante. Como era normal, al poco tiempo los tres fueron exaltados al siguiente grado masón, el de «compañero», hecho que tuvo lugar al comienzo del verano, el día 6 de julio, pero solamente las dos hermanas ascendieron en su grado de compromiso y dedicación a la logia, y en el mes de agosto se convirtieron en «maestro masón» o grado 3, algo que nunca consiguieron ni Juana ni Dolores. El motivo se desconoce, pero Manuel siguió siendo «compañero» un año más, aunque eso no fue obstáculo para que, cuando en diciembre de 1887 se eligieron los cargos directivos de la Logia para el siguiente periodo, él y su hermana Ana fueran designados «Secretarios Adjuntos», y Amalia «Oradora Adjunta».

Los jóvenes que vivían en la portería de la Facultad se debieron señalar socialmente con total seguridad, pero especialmente las dos hermanas, y, por ser mujeres aún más, tuvieron que ser la «comidilla» de la Facultad y del barrio de Hércules. Y más aún cuando, casi de forma paralela a su entrada en la masonería, se unieron al Círculo Librepensador «Guillén Martínez» que había ayudado a fundar el ya entonces mítico Fermín Salvochea que había vuelto a Cádiz indultado tras la muerte de Alfonso XII. Sus apariciones en el Círculo, especialmente la de Amalia Carvia, con sus lecturas de poesía, prosa y algún discurso, aumentarían aún más su notoriedad $^{7}$. A lo que hay que añadir la circunstancia de que hacía dos años que había empezado a publicar sus trabajos en conocidos periódicos librepensadores de Madrid y Barcelona ${ }^{8}$.

6 Flecha García, C. y Delgado Fernández, A. Alumnas del Instituto de Segunda Enseñanza de Cádiz antes de 1910. Tavira: Revista de ciencias de la educación, n. ${ }^{\circ}$ 13, 1996, pp. 25-42.

7 Almisas Albéndiz, M. ¡Paso a la mujer! Biografia de Amalia Carvia. El Puerto (Cádiz): Ed. Suroeste, 2019.

8 CARVIA, A. Desde las cumbres. Obra recopilada y editada por Manuel Almisas Albéndiz. Ed. Suroeste, 2019. DOI del artículo:

https://doi.org/10.25267/Cuad investig fondos arch UCA.2020.i2.01

Editorial

REVISTAS | Universidad de Cádiz 
Siguiendo con la trayectoria masónica de los hermanos Carvia Bernal, en octubre de 1888 Manuel fue exaltado finalmente al grado 3, «maestro masón», y en diciembre fue elegido para el importante cargo de «Secretario General» de la Logia «Regeneración». Mientras que Ana siguió siendo la Secretaria Adjunta estos años, y Amalia la Oradora Adjunta.

Ana acabó el Bachillerato con unas calificaciones brillantísimas -con un Sobresaliente global-, y en el curso 1889-1890, junto a la que será la primera médica licenciada en la Facultad de Medicina de Cádiz, Antonia Monreal Andrés, se matriculó en 1. ${ }^{\circ}$ de Alemán en el Instituto Provincial y en las asignaturas de Física, Química, Zoología y Mineralogía y Botánica del Curso o Año Preparatorio 9 para cursar la licenciatura de medicina. Sin embargo, en su expediente personal no constan las calificaciones de estas materias porque Ana tuvo que abandonar los estudios debido a la precariedad económica de la familia. Ese es el motivo que ella misma alegará años más tarde y por el que Ana Carvia no llegó nunca a matricularse en Medicina. Porque cualidades no le faltaban. Amalia siempre elogió a su hermana pequeña, describiéndola como muy inteligente y estudiosa, y la prueba lo tenemos en su expediente de Bachillerato, donde destacaba su nota final de Sobresaliente, y eso que tuvo que estudiar, por ser mujer, en la modalidad «doméstica», sin poder pisar las aulas. Su hermano Manuel, en cambio, solo superó el Bachillerato con un «Aprobado».

Figura 2. Expediente personal de Ana Carvia Bernal. Matrícula en Año Preparatorio para la Facultad de Medicina, septiembre de 1889.

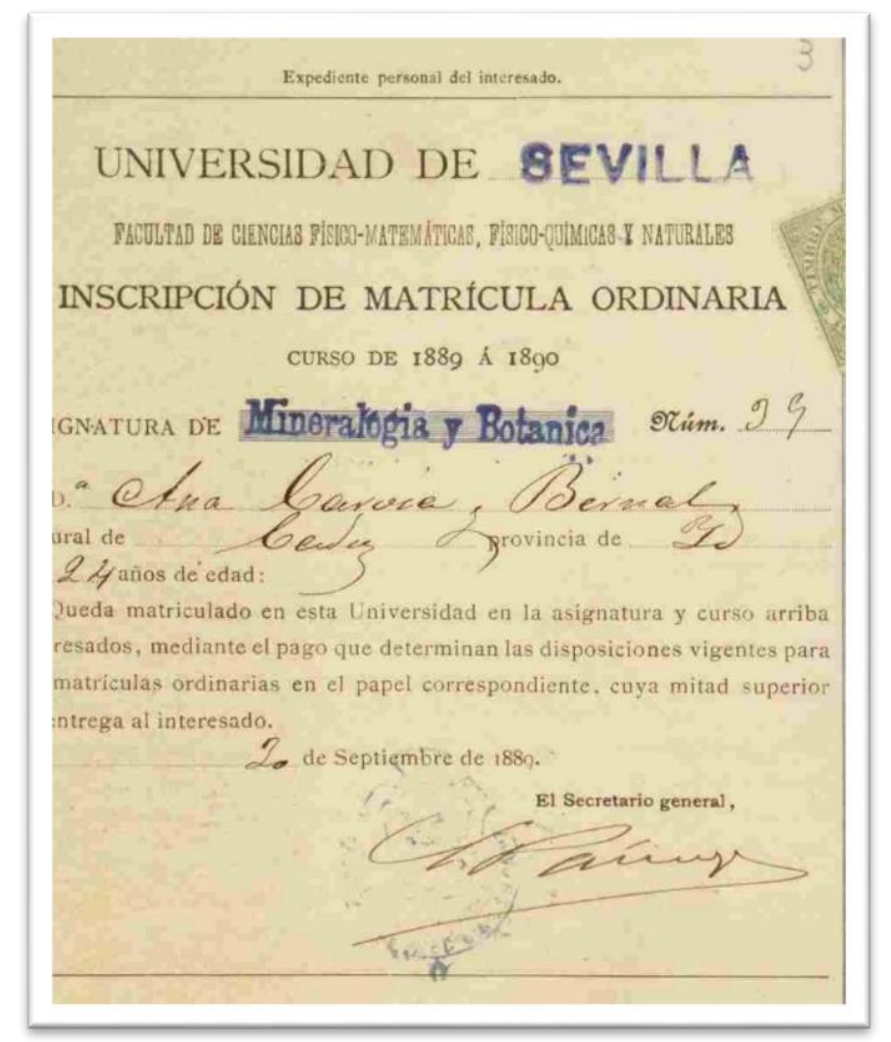

Fuente: Archivo de la Universidad de Cádiz (AUCA)

9 Archivo de la Universidad de Cádiz (AUCA). C-155 (4)-2 FM, expediente personal de Ana Carvia. DOI del artículo: https://doi.org/10.25267/Cuad investig fondos arch UCA.2020.i2.01

Editorial REVISTAS | Universidad de Cádiz 


\section{La familia Carvia Bernal y la Facultad de Medicina. Una historia de librepensamiento y masonería en el Cádiz de finales del siglo XIX}

En mayo de 1889, a los dos años de ingresar en la Logia «Regeneración», a Manuel se le concedió la «Plancha de quite» (separación definitiva de la logia) que había solicitado y esta decisión solo puede explicarse por la inminencia de su graduación y sus posibles expectativas de una vida profesional lejos de Cádiz. Lo cierto es que Manuel dejó el puesto de «Secretario general» de la Logia a su hermana Ana, cargo inusual para una mujer en las logias masonas de Cádiz en aquellos años de fin de siglo, designación directiva que se hizo oficial en las elecciones del mes de diciembre.

En ese mismo año 1889, la familia Carvia Bernal dejará la casa-portería de la Facultad después de catorce años, al ascender Manuel Carvia Carneiro en el escalafón laboral y convertirse en uno de los dos bedeles de la Facultad junto a Andrés Longo Lorenzo, que ya había sido también antiguo portero de la Facultad. El nombramiento de «bedel $2^{\circ} »$ tuvo lugar el 31 de octubre ${ }^{10}$ y a partir de entonces la familia se mudó a la vecina calle o plazuela de Jesús Nazareno n. ${ }^{\circ}$ 9-11, donde residirá más de una década.

En el expediente personal de Manuel consta que consiguió el Grado de Licenciatura en Medicina a finales de 1890, tras un suspenso en el primer ejercicio de Grado en la convocatoria de junio.

Poco después, Manuel Carvia Bernal abandonó Cádiz para siempre, ocupando el puesto de «médico cirujano» en los pueblos gaditanos de Algodonales y El Gastor, y estableciéndose más tarde, y definitivamente, en el malagueño Cortes de la Frontera, donde fundó una familia y falleció en 1932.

Así que Manuel Carvia Carneiro, uno de los dos bedeles de la Facultad, se quedó viviendo con sus dos hijas masonas, cada vez más involucradas en los círculos librepensadores y republicanos de la ciudad y ejerciendo esporádicamente de maestras laicas. Y, sobre todo, la figura de Amalia, su hija mayor, fue destacando como oradora y escritora, hasta convertirse en la mujer más influyente de los círculos progresistas de la ciudad, además de como asidua «publicista» en diarios locales como «El Manifiesto» o «El Pueblo».

En esta época Amalia sufrió críticas y persecución mediática por parte de los estamentos ultracatólicos y «jesuíticos» de Cádiz, cosa que afectaría a toda la familia. De hecho, ella misma escribirá un artículo en el que lamentaba que una amiga íntima desde la infancia hubiera roto con ella porque había descubierto que era «librepensadora» ${ }^{11}$. Y también años más tarde, desde las páginas de El Pueblo de Cádiz le escribía irónicamente a una jesuita que había mostrado deseos de conocerla, en el momento cumbre de su «popularidad» ${ }^{12}$.

Pero lo que ya tuvo que ser el «acabose» de la fama de las hijas del bedel de la Facultad de Medicina fue cuando en abril de 1895 fundaron la única logia femenina que ha existido en

10 Archivo de la Universidad de Cádiz (AUCA). Libro Registro de Títulos de la Facultad de Medicina en Cádiz, 1885-1918. L-279 FM. Anotación 282, 1889.

11 Carvia, A. Reflexiona, en Las Dominicales del Libre Pensamiento (Madrid) de 13 de junio de 1891. Recogido en Desde las Cumbres (Recopilación de su obra periodística). Obra recopilada y editada por Manuel Almisas Albéndiz. El Puerto (Cádiz): Editorial Suroeste, 2019.

12 Carvia, A. A una jesuita, en El Pueblo (Cádiz) de 18 de mayo de 1899. Recogido en Desde las Cumbres (Recopilación de su obra periodística). Obra recopilada y editada por Manuel Almisas Albéndiz. El Puerto (Cádiz): Editorial Suroeste, 2019.

DOI del artículo:

https://doi.org/10.25267/Cuad investig fondos arch UCA.2020.i2.01 
Cádiz, a la que llamaron «Hijas de la Regeneración», y de la que la propia Amalia fue elegida su Venerable Maestra durante tres años. Es de destacar que, en esos años, tuvieron una especie de «doble militancia», pues seguían participando en las reuniones («tenidas») de la logia mixta «Regeneración», e incluso Amalia fue elegida para participar en la Federación Provincial Masónica gaditana, y formó parte de su Comisión Ejecutiva desde abril de 1896, hecho del todo inusual y sobresaliente, como puede comprenderse.

Figura 3. Aspecto de Amalia Carvia Bernal en 1896, cuando era escritora, librepensadora y «Venerable Maestra» en Cádiz.

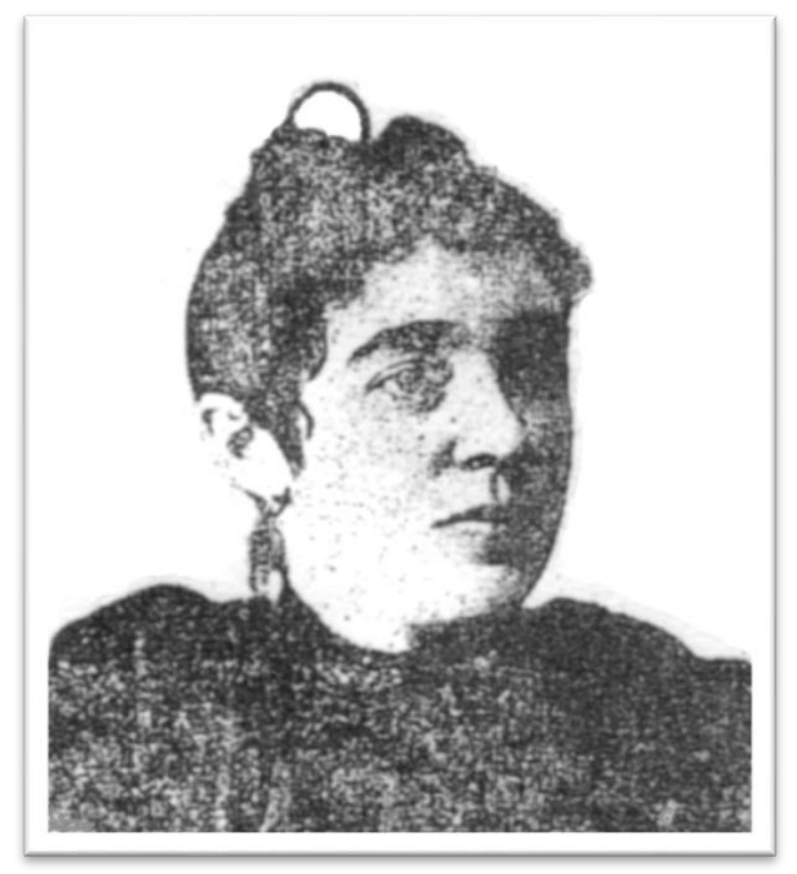

Fuente: Portada de La Conciencia Libre (Valencia) del 1 de enero de 1897, número 2 extraordinario.

Esta historia está llegando a su fin. Manuel Carvia Carneiro, ya viudo desde hacía unos años y viviendo sin sus hijos, pues su hija pequeña Ana se había marchado a Valencia en 1897, a la que seguiría Amalia en 1900, alcanzó el puesto de Conserje en junio de $1900^{13}$, cuando llevaba 25 años trabajando para la Facultad de Medicina y vivía ahora en la cercana calle Soledad.

Aun cuando sus dos hijas se marcharon a Valencia ya sin pertenecer a la masonería, durante un tiempo sus antiguos correligionarios y compañeras de la logia y de la Asociación Femenina «Concepción Arenal» que Amalia había fundado, las siguieron recordando y es posible que, a pesar de la grave crisis que padeció el mundo masón tras las pérdidas de las colonias de Cuba, Puerto Rico y las Filipinas en 1898, todavía recordarían a Manuel como «el padre de los masones de la Facultad».

A Manuel Carvia Carneiro le alcanzó la muerte luciendo su lujoso uniforme de Conserje de la Facultad. Fue en 1908 y estaba a punto de jubilarse.

13 Archivo de la Universidad de Cádiz (AUCA) Libro de Títulos de la Facultad de Medicina, 1900, anotación 43.

DOI del artículo:

https://doi.org/10.25267/Cuad investig fondos arch UCA.2020.i2.01 


\section{FUENTES DOCUMENTALES:}

Archivo de la Universidad de Cádiz (AUCA).

Archivo del Centro Documental de la Memoria Histórica (CDMH) de Salamanca.

\section{REFERENCIAS BIBLIOGRÁFICAS:}

Almisas Albéndiz, M., 2019 ¡Paso a la mujer! Biografía de Amalia Carvia. El Puerto de Santa María (Cádiz): Editorial Suroeste.

CARviA, A., 2019. Desde las cumbres, obra periodística y literaria recopilada y editada por Manuel Almisas Albéndiz. El Puerto de Santa María (Cádiz): Editorial Suroeste.

ENRÍQUEZ DEL ÁRBOL, E. La Masonería en Andalucía en el último tercio del S. XIX. En: JosÉ Antonio Ferrer Benimeli (Coord.), La Masonería en la España del siglo XIX, Consejería de Educación y Cultura de la Junta de Castilla y León, Volumen I, año 1987, pp. 217-245.

FERnÁNDEZ LuCEÑO, M. ${ }^{\mathrm{a}}$ V., 2016. Médicos republicanos y masones en Andalucía contemporánea. La represión franquista. Sevilla: Editorial Aconcagua.

Flecha García, C. y Delgado Fernández, A., 1996. Alumnas del Instituto de Segunda Enseñanza de Cádiz antes de 1910. Tavira: Revista de ciencias de la educación, n. ${ }^{\circ} 13$, pp. 25-42. 